\title{
Assignment of Tangier disease to chromosome $9 q 31$ by a graphical linkage exclusion strategy
}

Stephan Rust et al.

Nature Genetics 20, 96-98 (1998).

Due to a printing error, accurate reproduction of Figure 2 was compromised. The correct version is printed below. Corrected colourreprints are available from the authors

Fig. 2 Traffic-light evaluation of results. a, Testing of sibpairs for shared segments showing IBD. Pedigree members are identified as in Fig. 1. As an example of our data analysis, the results from the two affected sibpairs E121/E122 and A11/A12 are shown. Markers were from the ABI Prism linkage mapping set (T16-1 =D9S283, H14-4=D9S287, H14-3=D9S279, F16-4=D9S290). For clarity, only the chromosome transmitted from the affected parent is shown for each sib. Data are evaluated in the column 'shared segment' for segments IBD, but using colours: E121/E122 share the segment [H14-3-F16-4] IBD, indicated by a solid frame surrounding the segments, and the corresponding region in the column 'shared segment' is labelled green (localization of the TD gene allowed). They do not share the segment [T16-1-H14-4]. The corresponding segment is labelled red (localization of the TD gene not allowed). In the analysis of sibpair A11/A12, marker H14-4 was not informative due to homozygosity in the father. This is indicated by a dashed frame and a yellow (undefined) segment in the column 'shared segment'. In the column 'IBD-sum', the combined results are shown with each marker locus being excluded due to the previous IBD-tests. Yellow arrowheads highlight the region between the coloured segments, where the bars are interrupted and where we had no information on the IBD-status due to crossovers in that region. b. Columns I and II depict the traffic-light results from the 'shared segment' columns of (a) drawn to scale showing the distances between the markers in CM. In this presentation, the critical crossover region, highlighted in (a) by yellow arrowheads, is now coloured yellow, indicating that the region may be IBD. Column III provides the summary of all affected pair comparisons and is a simple alternate sequence of red and yellow regions, where yellow indicates a need for further analysis. Therefore, the additional markers T70-3=D9S277, F73-01=D9S53, F72-70=D9S1784, F71-15=D9S1832, F71-14=D9S1801 and F72-82 =D9S1677 were analysed. Columns IV and V show the corresponding results for the analysis of sibpairsE121/E122 and A11/A12. Column VI shows the overall results from all sibpairs, which were obtained by labelling red all those segments which were labelled red in at least one sibpair analysis. In the summary bar (column VI) only those regions in which no red occurred in any sibpair analysis are labelled green. Column VII shows the position of a heterozygous deletion in chromosome 9 in an individual with a heterozygous low HDL phenotype. All marker loci shown were typed in the patient and his parents. The patient obtained one allele from each parent at H14-4 and F16-4 and had two different alleles at these positions. These markers are labelled red to indicate the outer limit of the deletion. Whereas the parents had four different alleles at both the loci T70-3 and H14-3, the patient did not obtain an allele from his father at these loci; this also applied to the intervening markers. The deleted region is shown blank. $a$

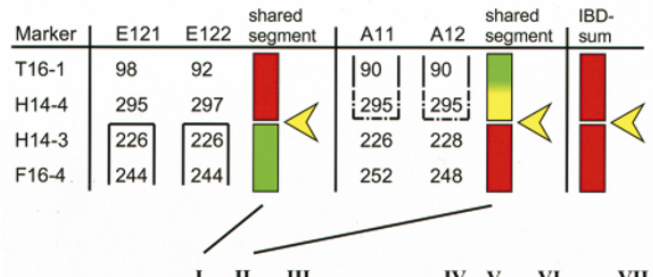

\section{ค}

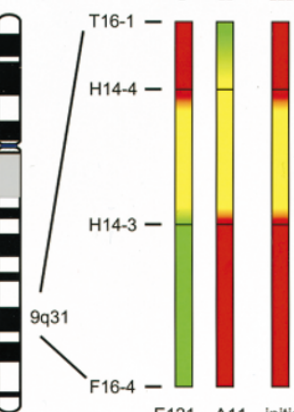

E121 A11 initia

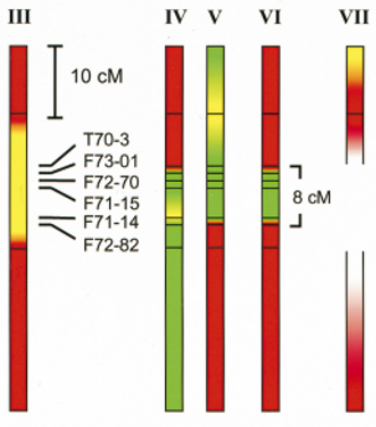

E121 A11 final E122 A12 Sum

\section{Inversin, a novel gene in the vertebrate left-right axis pathw ay, is partially deleted in the inv mouse}

\section{David Morgan et al.}

Nature Genetics 20, 149-156 (1998).

Due to a printing error, accurate reproduction of Figure 1 was compromised. The correct version is printed below.

Fig. 1 Genomic organization of the mouse Invs gene and CDNA contig assembly. a, Schematic of wild-type and inv chromosome 4. Transgene insertion involved integration of two copies of a tyrosinase minigene, deletion of a 47-kb sequence and duplication of a short segment initially located $6 \mathrm{cM}$ distal to the region that was deleted. b, Organization of YAC and $\mathrm{BAC}$ dones spanning the region deleted in the inv mouse. A, Apal; B, BssHII; E, Eagl; M, Mlul; N, Notl; Na, Narl; Nh, Nhel; S, Smal; X, Xhol. Sequence contigs 82/2145 and 1908 are derived from clone BAC394-P2, which spans the deleted region. c, Exon/intron organization of the Invs gene spanning the deleted region. d, cDNA contig assembly. Vertical lines in the bar at top indicate position of exon boundaries in the CDNA sequence and horizontal lines below indicate individual CDNA clones. Clones obtained by RT-PCR and RACE are indicated by the prefix 'RT' or 'RA', respectively, and were obtained using mouse adult liver CDNA; sources of clones obtained by standard CDNA library screening are as follows: $\mathrm{Cd}$, Clontech mouse adult liver; pKE15-5, E15.0 mouse kidney; 583066 , Soares 2NbMT mouse thymus; and 636902, Soares NbMLN mouse lymph cDNA. Alternative splicing in exon 13 to give transcripts lacking a 360- or a 495-nt sequence (Fig. 5) is indicated by an inverted V. ATG, translation initiation codon; Ter, stop codon; pA1, pA2, alternative polyadenylation signals.
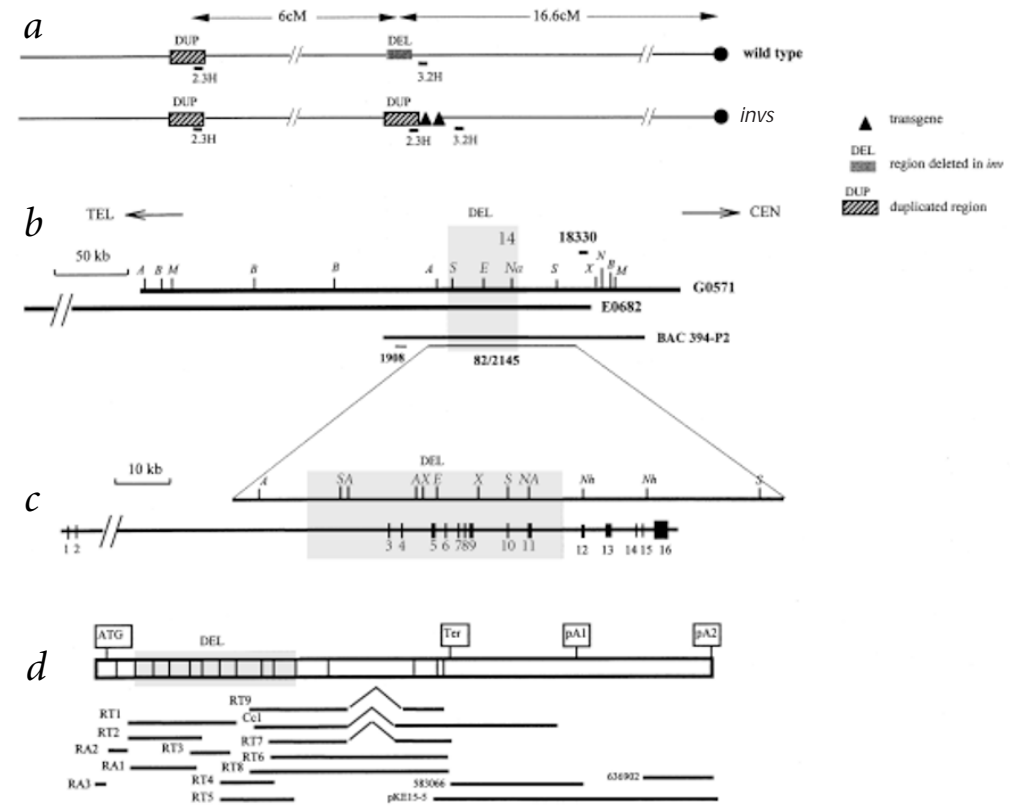